\title{
A Velociraptor wishbone
}

The 'wishbone' of birds comprises two clavicles fused into a structure ${ }^{1}$ known as a furcula. In an influential 1926 book on bird origins by Heilmann ${ }^{2}$, the furcula's supposed absence in dinosaurs was considered powerful evidence barring them from bird ancestry. A furcula has now been found in several theropod dinosaurs ${ }^{3,4}$, but its absence in other theropods, and the uncertainty of whether this absence is real or an artefact of preservation, obscures the evolutionary history of this structure ${ }^{5}$. Here we report the discovery of a furcula in Dromaeosauridae, a group posited to be the closest relative of birds ${ }^{6,7}$.

Among close bird relatives (non-avialian Maniraptora), a furcula is known only in Oviraptoridae $^{8}$. Although a furcula was tentatively reported to be present in an articulated skeleton of Velociraptor mongoliensis preserved embracing a Protoceratops skeleton $^{3,6}$, the only firsthand description of this specimen maintained that it is not present ${ }^{8}$, and our observations of that specimen confirm this. A furcula has not been identified in any of the other five described species of Dromaeosauridae. Other taxa either lack any evidence of clavicles or, in one troodontid specimen $^{9}$, clavicles are present but not fused. But the rarity of articulated specimens, the similarity of the furcula to ribs and gastralia, and incomplete preservation conspire to obscure whether this absence is real.

A partial skeleton of Velociraptor mongoliensis (specimen IGM 100/976) was discovered at Tugrugeen Shireh, Mongolia, during the 1991 expedition of the Mongolian Academy of Sciences-American Museum of Natural History Expedition. The specimen consists of a nearly complete skull and the anterior part of the skeleton, preserving derived characters that allow definitive reference to Velociraptor mongoliensis ${ }^{10}$.
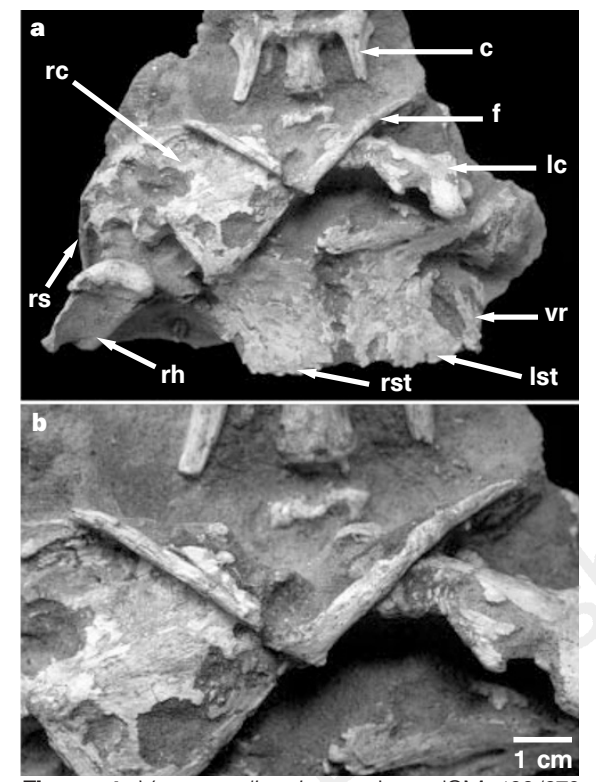

Figure 1 V. mongoliensis specimen IGM 100/976 with ' $V$ '-shaped furcula. a, Ventral view of anterior trunk region. c, posterior cervical vertebrae; f, furcula; Ic, left coracoid; vr, ventral ribs; Ist, left sternal plate; rst, right sternal plate; rh, right humerus; rs, right scapula; rc, right coracoid. b, Detail of same region.

The bones are heavily bioturbated, and many elements are punctured by borings similar to those often present in Djadokhta Formation sediments ${ }^{11}$, but the specimen is otherwise very well preserved and articulated. The block that holds the furcula also preserves a pair of sternal plates, posterior cervical vertebrae, the proximal ends of both humeri, the coracoids and the right scapula. The specimen is large relative to others of this species, and the cervical ribs are nearly fused to the vertebral centra, indicating that the specimen is near full-size.

The furcula is in articulation between the scapulocoracoids anterior to the sternal plates (Fig. 1), in the same position as in oviraptorids and birds. The bone is ' $\mathrm{V}$ 'shaped and very slender, much thinner than in oviraptorids and Archaeopteryx. In crosssection it is nearly circular. A short midline process, the hypocleidium, extends posteroventrally. The proximal, or epicleideal, process sweeps posterodorsally to articulate with the acromion of the scapulocoracoid. The proximal process of the furcula tapers to a point where it contacts the scapulocoracoid, and the posterior articulating surface contacting the acromion is smooth.

The broad distribution of a furcula among the non-flying relatives of birds $s^{4,5,8,12}$ indicates that its origin is not tied to the origin of flight. The furcula's use in powered avian flight is therefore a co-option of a structure already present before flight evolved in the lineage leading to modern birds $^{4}$. The discovery of a furcula in a taxon previously known from an articulated specimen underscores the difficulty of assessing whether the apparent absence of this feature is real or an artefact of preservation or ontogenetic sampling 5 .

Mark A. Norell, Peter Makovicky

Department of Vertebrate Paleontology,

American Museum of Natural History,

79th Street@Central Park West,

New York, New York 10024-5192, USA

James M. Clark

Department of Biological Sciences,

George Washington University,

Washington DC 20052, USA

1. Baumel, J. J. \& Witmer, L. M. Publ. Nuttal Ornithol. Club 23,
45-132 (1993).
2. Heilmann, G. The Origin of Birds (Witherby, London, 1926).
3. Ostrom, J. Biol. J. Linn. Soc. 8, $91-182$ (1976).
4. Makovicky, P. J. \& Currie, P. J. J. Vert. Paleontol. (in the press).
5. Bryant, H. \& Russell, H. J. Vert. Paleontol. 13, 171-184 (1993).
6. Gauthier, J. A. Calif. Acad. Sci. Mem. 8, 1-55 (1986).
7. Holtz, T. J. J. Paleontol. 70, 536-538 (1996).
8. Barsbold, R. Sovmestnaya Sovetsko-Mongol'skaya Paleontol.
Ekspiditsiya, Trudy 19, 5-119 (1983).
9. Russell, D. A. \& Dong, Z.-H. Can. J. Earth Sci. 30, 2163 (1993).
10. Norell. M. A. et al. J. Vert. Paleontol. 12 Suppl. 45A (1992).
11. Jerzykiewicz, T. et al. Can. J. Earth Sci. 30, 2180-2195 (1993).
12. Chure, D. J. \& Madsen, J. H. J. Vert. Paleontol. 16, 573-577 (1996).

crystals obtained have found little practical application, largely because the colloidal crystals are formed in liquid environments and either do not survive drying or have poor mechanical stability. Our scheme overcomes these problems by replicating the structure with a stable silica matrix.

Formation of colloidal crystals from monodisperse colloid particles is easily achieved by increasing their volume fraction in the vicinity of a flat wall ${ }^{12}$. We filtered polystyrene latex microspheres (size range 200-1,000 nm), carrying either negative surface charges (sulphate) or positive charges (amidine), in suspension through a smooth narrow-pore membrane. When the particulate layers had been deposited, they could be washed and penetrated by different liquid 


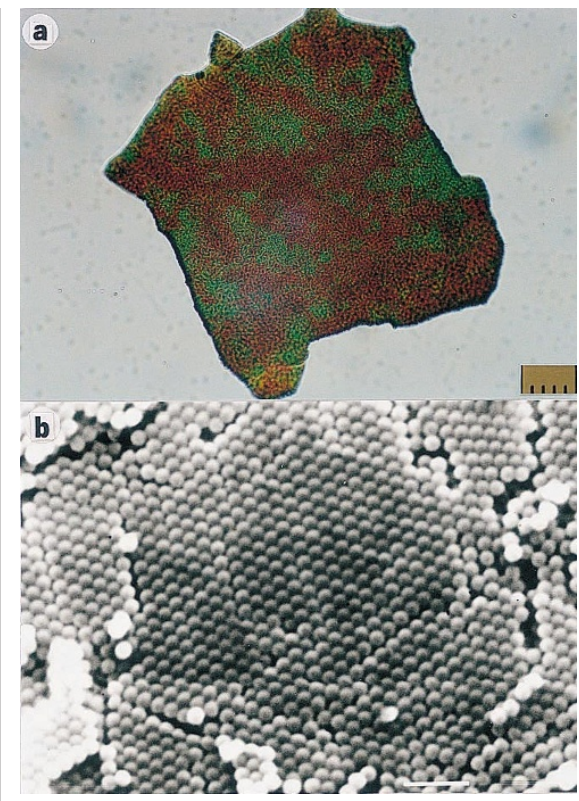

Figure 1 Micrographs of ordered latex templates. a, Optical microscopy in transmitted light. The different colours probably correspond to crystal domains of different symmetry or orientation. $\mathbf{b}$, Scanning electron micrograph (SEM). Ordered latex layers display either hexagonal or cubic packing. Scale bars: $50 \mu \mathrm{m}$ and $1 \mu \mathrm{m}$, respectively.

media. The latex particles accumulated slowly on the membrane surface, building up closely packed, ordered layers roughly $10 \mu \mathrm{m}$ thick. The deposited crystalline layer could be broken and detached from the membrane surface for analysis by light and scanning electron microscopy (Fig. 1).

To induce silica polymerization the microsphere surfaces had to be functionalized in situ by adsorption of the surfactant hexadecyltrimethyl ammonium bromide (HTAB). We soaked the crystalline latex layers with $0.02 \mathrm{M}$ HTAB solution for $20 \mathrm{~min}$, then removed the excess unadsorbed surfactant by washing briefly with deionized water. We mineralized the cavities in the arrays by passing $0.5 \mathrm{M}$ silica solution through the latex-covered filter. The permeability of the layers decreased as the polymerization process continued, so that flow through the filter stopped in less than one minute. When the silica solution had gelled inside the colloidal crystal layer, we removed the excess solution and dried the latex/silica composite under vacuum. The latex templates inside the polymerized silica were removed by heating at $450{ }^{\circ} \mathrm{C}$ for $4 \mathrm{~h}$, leaving silica flakes of very low density as the final product.

Scanning electron microscopy shows that the material is built up of three-dimensional ordered arrays of uniform pores. Examples of the discrete morphology of the material are shown in Fig. 2. By varying the size of the latex microspheres used, we were able to produce organized materials with pore sizes ranging from about $150 \mathrm{~nm}$ to

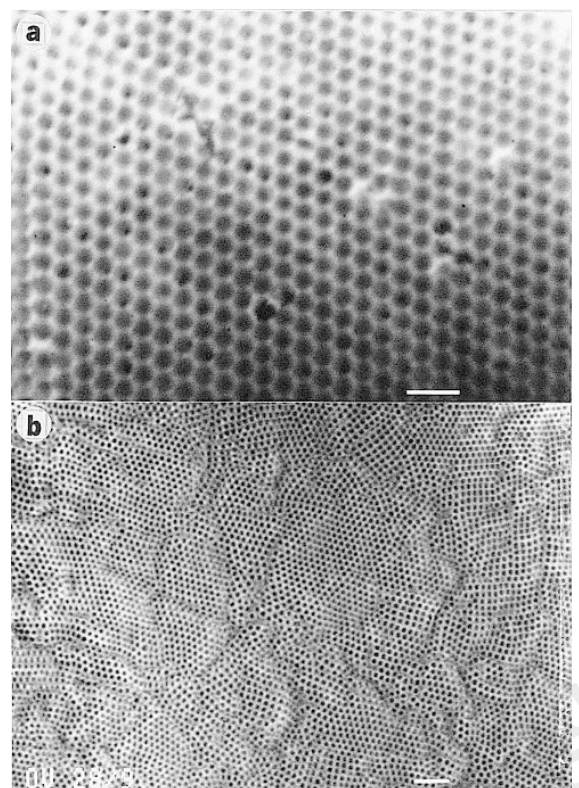

Figure 2 SEM of the microporous silica structures. Latex particles of $\mathbf{a}, 560 \mathrm{~nm}$ and $\mathbf{b}, 300 \mathrm{~nm}$ diameters were used as templates. Large ordered arrays of spherical cavities, representing a negative replica of the original colloidal crystal embedded in the silica, are seen. The silica flakes appear to be built up of many similar domains with different crystal orientations. Details of materials and methods are available on request from the authors. Scale bars, $1 \mu \mathrm{m}$.

$1 \mu \mathrm{m}$. A comparison between the repeat units of the silica replicas and the original latex crystals showed that the baked materials had shrunk by $20-35 \%$, a value that is higher than, but comparable to, that in the M41S mesoporous silicas ${ }^{4}$.

Our results show that it is possible to obtain highly structured silica materials in which the pore size, shape and ordering can be precisely controlled within a wide range that has previously been unattainable. The method is powerful and controllable, and could be adapted for large-scale production.

\section{O. D. Velev, T. A. Jede}

\section{R. F. Lobo, A. M. Lenhoff}

Department of Chemical Engineering,

University of Delaware, Newark,

Delaware 19716, USA

e-mail:velev@che.udel.edu

1. Kresge, C. T., Leonowicz, M. E., Roth, W. J., Vartuli, J. C. \& Beck, J. S. Nature 359, 710-712 (1992).

2. Beck, J. S. et al. J. Am. Chem. Soc. 114, 10834-10843 (1992).

3. Monnier, A. et al. Science 261, 1299-1303 (1993).

4. Raman, K. N., Anderson, M. T. \& Brinker, C. J. Chem. Mater. 8, 1682-1701 (1996)

5. Davis, S. A., Burkett, S. L., Mendelson, N. H. \& Mann, S. Nature 385, 420-423 (1997).

6. Mann, S. \& Ozin, G. A. Nature 382, 313-318 (1996).

7. Arora, A. K. \& Rajagopalan, R. in Ordering and Phase Transitions in Charged Colloids (eds Arora, A. K. \& Tata, B. V. R.) 1-13 (VCH, New York, 1996).

8. Larsen, A. E. \& Grier, D. G. Nature 385, 230-233 (1997).

9. Trau, M., Saville, D. A. \& Aksay, I. A. Science 272, 706-709 (1996)

10. Denkov, N. D. et al. Nature 361, 26 (1993).

11.van Blaaderen, A., Ruel, R. \& Wiltzius, P. Nature 385, 321-324 (1997)

12. Davis, K. E., Russel, W. B. \& Glantschnig, W. J. Science 245, 507-510 (1989).

\section{The same prion strain causes vCID and BSE}

Epidemiological and clinicopathological studies, allied with pathological prion protein $\left(\mathrm{PrP}^{\mathrm{Sc}}\right)$ analysis, strongly support the hypothesis that the human prion disease new variant Creutzfeldt-Jakob disease (vCJD) is causally related to bovine spongiform encephalopathy (BSE) $)^{1,2}$, but considerable controversy remains. Distinct prion strains are distinguished by their biological properties on transmission to laboratory animals and by physical and chemical differences in $\mathrm{PrP}^{\mathrm{Sc}}$ strains. We now find that the biological and molecular transmission characteristics of vCJD are consistent with it being the human counterpart of BSE.

We studied transgenic mice expressing only human $\operatorname{PrP}\left(\mathrm{HuPrP}^{+/+} \mathrm{Prn}-p^{\mathrm{o} / \mathrm{o}}\right)$, which have been shown to lack a species barrier to human prions from one iatrogenic CJD case $^{3}$, comparing them with non-transgenic (FVB) mice. All of 16 further CJD cases, encompassing a wide range of clinicopathological phenotypes, all three $\operatorname{PrP}^{\mathrm{Sc}}$ types reported in sporadic and acquired prion diseases $^{2}$ and all PRNP genotypes at polymorphic codon 129 , a key determinant of genetic susceptibility to human prion diseases ${ }^{4-6}$, were transmitted to these transgenic mice.

Almost all inoculated transgenic mice contracted disease with similar short incubation periods, consistent with a lack of species barrier to these isolates (Table 1). These transgenic mice express human $\operatorname{PrP}$ homozygous for valine at codon 129 . However, there was no significant difference in mean incubation periods between inocula of the different codon 129 genotypes. $\mathrm{PrP}^{\mathrm{Sc}}$ typing of these transmissions showed that the same prion types seen in sporadic and iatrogenic CJD (types 1-3) are produced, distinct from that seen in vCJD (type 4$)^{2}$. Only occasional transmissions, at longer and variable incubation periods, were seen in FVB mice.

In contrast, efficient transmission of vCJD to FVB mice was observed (Table 1) although incubation periods were prolonged. Conversely, the attack rate of vCJD in the transgenic mice was reduced in comparison to typical CJD, and incubation periods were generally more variable and prolonged. Mean incubation periods to these six vCJD cases were similar in both types of mice. The clinical course in vCJDinoculated transgenic mice was much longer than in transmissions of typical CJD. vCJD in humans is also associated with a long clinical duration ${ }^{1}$. Some mice, as well as showing typical neurological features, persistently walked backwards. This unusual clinical sign was not seen in transmissions of typical CJD, fatal familial insomnia or other 